\title{
Aproximación al estudio de las haciendas locales bajo Carlos III: Los propios del concejo murciano
}

\author{
F. Javier Guillamón y J. Perez-Hervás*
}

Las haciendas locales se nutrian principalmente de las aportaciones que les proporcionaban los propios y arbitrios. Éstos se constituian en "auténticos pilares" de las arcas municipales '. Sin embargo se precisa la distinción y aclaración de ambos conceptos, que siempre en la terminología del Antiguo Régimen aparecen englobados: como propios se entendía a aquellos elementos ya inmuebles, ya variables, que formaban parte del patrimonio concejil, en tanto que como arbitrios debe entenderse aquellos impuestos indirectos que recaían sobre el tráfico de los géneros comerciales -comestibles, esencialmente-, y cuyo origen, generalmente, se encontraba en alguna disposición de carácter transitorio que la monarquía hacia a favor de la ciudad y para un fin determinado, aunque a veces se prolongase sine die su aplicación. A pesar de esta distinción, los rendimientos de ambos apartados se computaban en conjunto y su utilización era indistinta, a pesar de las circunstancias previstas en las disposiciones que otorgaban el privilegio de cobrar dichos arbitrios $^{2}$.

No vamos a introducirnos en la cuestión del origen y desarrollo de los propios. Canga Argüelles y Alejandro Nieto, entre otros, nos han apor-

* Universidad de Murcia.

- Pablo Fernandez Albaladejo: “Monarquía ilustrada y Haciendas locales en la segunda mitad del siglo XVil!", en Estudios de Historia. De Ensenada a Mon, Ed. de M. Artola y L. M. Bilbao. Madrid 1984, p. 166.

${ }^{2}$ Bajo Felipe $V$ y Fernando VI se promulgaron reales facultades concediendo a la ciudad de Murcia el derecho de imponer arbitrios sobre la carne, el vino y el pescado. Sus fechas: $5-12-1726,1-10-1745$ y $21-5-1757$. 
tado interesantes estudios sobre el tema ${ }^{3}$. Lo que nadie pone en duda es que el fin primordial de la existencia de propios y de arbitrios era el de proporcionar rentas a las arcas municipales.

Durante la primera mitad del siglo XVIII la recién instaurada monarquía borbónica dictaría normas que por su significado pueden inscribirse en un contexto innovador y reformista dentro de la dinámica fiscal que la monarquía había desarrollado respecto a los bienes concejiles. La Instrucción de 1745, mandada por el Real y Supremo Consejo de Castilla para la intervención y administración y recaudación de arbitrios del Reino ${ }^{4}$, no era sino la culminación de un proceso que se venía gestando desde tiempos de Campillo como Secretario de Hacienda, firme partidario de un sistema de administración directa por parte del Estado ${ }^{5}$. A esta Instrucción de 1745 siguen la Ordenanza de Intendentes (1749), la Provisión de 1751 y la Instrucción de 1760, ya bajo Carlos III, la cual expresa desde el mismo preámbulo la preocupación por la buena gestión económica de los caudales municipales. En esta Instrucción de 30 de julio de 1760 aparece la figura de la Contaduría General de propios y arbitrios, órgano rector, por delegación del Consejo de Castilla, con poderes sobre las Juntas locales de Propios y Arbitrios.

Desde la Contaduría General, Manuel Becerra había enviado una serie de informes, sobre todo a partir del año 1763, sobre el comportamiento de las haciendas locales. Paralelamente, cada Municipio elaboraba su reglamento de propios y arbitrios. En 1765 la cifra de corporaciones que contaban con reglamento propio superaban a los 5.000 municipios; cuatro años más tarde, en 1769, la cantidad se habia duplicado llegando a 11.000 los pueblos que disponian de Reglamento ${ }^{6}$.

La Instrucción de 1760 marcaba las pautas a seguir en el ensamblamiento del mecanismo burocrático de centralización de propios y arbitrios hasta la desaparición de la Contaduría General, ya en 1836, por el Real Decreto de 12 de mayo. El Consejo recibia por esta Instrucción de

3 Véase J. Canga Arguelles: Diccionario de Hacienda, vol. II, p. 396, y A. Nieto: "Bienes Comunales". Rev. de Derecho Privado, Madrid 1964. También es interesante el art. de A. M. BERNAL: "Haciendas locales y tierras de propios: funcionalidad económica de los patrimonios municipales (siglos XVI y $x \mid x$ )". Hacienda Española, 55, 1978, pp. 285-312.

4 Para Murcia se aprobó la Instrucción por el Consejo de Castilla el día 22 de enero de 1746 (Archivo Histórico Municipal de Murcia, en adelante A.H.M.M., legajo-libro 301).

5 M. BitAR LetAYF: Economistas españoles en el siglo Xvim, Madrid 1968, p. 116.

6 Archivo General de Simancas, Secretaria de Hacienda, leg. 432. 
1760 la obligación de reglar y dotar las obligaciones y cargas que deben soportar cada pueblo, haciendo hincapié en la necesidad de que hubiere algún sobrante para la redención de censos que gravaban las arcas municipales, o bien para aliviar los arbitrios ${ }^{7}$.

La actividad del Consejo fue eficadísima y no dejaba de emitir órdenes para el buen funcionamiento de las Juntas Municipales de Propios y Arbitrios. Su complejidad fue tal que el mismo Consejo se vio obligado, o mejor dicho, precisado, a recopilar las disposiciones que había ido dictando para facilitar su manejo. Parece que hubo una recopilación en 1773 , pero la que hemos manejado es la de $1803^{\circ}$.

Asi pues, el control de la economía municipal quedaba centralizado en el Consejo de Castilla, con una Contaduría General de Propios y Arbitrios como órgano de control operativo y amplio poderes sobre las Juntas Locales. Para el Consejo quedaba el poder decisorio final. Sobre éste, lógicamente, el Monarca.

Punto y aparte merece atención la figura del Intendente y su relación con el tema. Con las Ordenanzas de 1749 los intendentes provinciales van a entender de propios y arbitrios, en tanto y cuanto tenían la obligación de supervisar el estado de las ciudades, pueblos y villas de su jurisdicción y, por consiguiente, la economía concejil. El Intendente está auxiliado por una Contaduría Provincial, que se ocuparía del control de las cuentas anuales de propios y arbitrios. Al mismo tiempo, el Intendente presidiría las Juntas Municipales de Propios y Arbitrios. La circular de 4 de julio de 1786 definiría las obligaciones del Intendente en el ámbito de los propios y arbitrios ${ }^{9}$.

La creación de los Diputados del Común y su participación en las Juntas servirian para acentuar el control sobre los propios y arbitrios concejiles, al tiempo que se reforzaba la independencia respecto a la corporación municipal.

\footnotetext{
${ }^{7}$ Nov. Rec. VII,16,13. Un auto de fecha 31 de octubre de 1761 desarrollaría este precepto.

${ }^{8}$ Hemos utilizado el libro de órdenes de 1803 . En el A.H.M.M., legajo n. ${ }^{\circ} 4.109$, se encuentra el libro de copias de órdenes recopiladas desde 1761 a 1803.

${ }^{9}$ La Instrucción de 21 de septiembre de 1785 reiteraba a los administradores de provincias la obligación de informarse sobre la naturaleza y estado de los propios y arbitrios.
} 
Murcia, concejo que había experimentado a lo largo de todo el siglo XVIII un crecimiento espectacular, va a contar desde el año 1762 con un Reglamento que va a poner en claro $-\mathrm{y}$ para lo sucesivo-, todo lo concerniente a Propios y Arbitrios ${ }^{10}$. Al lado de este Reglamento, el Archivo Municipal de Murcia guarda una extensísima y rica documentación sobre los bienes patrimoniales concejiles de tal forma que podríamos seguir año por año la problemática que nos ocupa, incluso hemos encontrado libros de actas de las reuniones de las Juntas de Propios y Arbitrios del concejo de Murcia ".

Para este artículo hemos seleccionado una serie de años para entender, aunque sea de una forma aproximativa, el comportamiento de los propios del Ayuntamiento de Murcia. Así, hemos escogido los años de 1700,1744 - perfectamente estudiado por la Dra. Cremades ${ }^{12}-, 1760$, 1799 y, ya dentro del siglo xIX, el año 1820. Como el lector podrá apreciar en el cuadro 1 son pocos los propios que aparecen en cada uno de los años escogidos en esta muestra. La bibliografía existente sobre el tema nos enseña que es casi imposible aplicar una misma tipologia para encasillar todo este completo "cajón de sastre", si se me permite la expresión, que constituye el aspecto de los propios de un ayuntamiento. No obstante he preferido arriesgarme a presentar los propios del Ayuntamiento de Murcia bajo un esquema definido que dejar sin ordenarlos y mostrarlos tal cual aparecen reflejados en los diferentes Reglamentos que conozco y he manejado. Así pues, entre el modelo de exposición que de los propios del Ayuntamiento de Salamanca hace Miguel Motta ${ }^{13}$, y el que presenta la Dra. Cremades Criñan para los propios del Ayuntamiento de Murcia ${ }^{14}$, hemos optado por el segundo, puesto que los parámetros utilizados para su confección están basados en la importancia de

10 Por suerte para el investigador, la documentación real existente en el A.H.M.M. sobre propios y arbitrios es abundantísima, no sólo en legajos, sino también en libros de cuentas, y Actas de reuniones de la Junta de Propios de la Ciudad de Murcia. El Reglamento de 1762 se encuentra en el legajo 1.534

11 A.H.M.M. legajos 2.700, 4.109, entre los más de 300 legajos y libros registrados. Estos que señalamos se refieren a la Junta del Pósito y libro de órdenes de Propios, por señalar algunos.

${ }^{12}$ La problemática de los propios y arbitrios para la primera mitad del siglo XVIII en Murcia ha sido publicada por la Dra. C. M. CREMADES GRIÑAN, bajo el nombre de Economía y Hacienda Local del Concejo de Murcia en el siglo xvII (1701-1759), Murcia 1986. También de la misma autora es el interesante librito Alimentación y consumo en la ciudad de Murcia durante el siglo xvIII (1701-1766), Murcia 1984.

13 J. I. Miguel-Motta: El municipio de Salamanca a finales del Antiguo Régimen. Contribución al Estudio de su organización institucional, Salamanca 1984, p. 178.

${ }^{14}$ C. M. Cremades Griñan: Economia y Hacienda local..., p. 274. 
los ingresos que proporcionaban cada mate y la naturaleza jurídica de los mismos.

\section{CUADRO 1}

LOS PROPIOS Y ARBITRIOS DEL CONCEJO MURCIANO EN EL SIGLO XVIII (1700-1820)

\begin{tabular}{|c|c|c|c|c|c|}
\hline CONCEPTO & 1700 & 1744 & 1760 & 1799 & 1820 \\
\hline A) PROPIOS MAYORES ${ }^{1}$ & & & & & \\
\hline Encañizada & SI & SI & SI & SI & $\mathrm{NO}^{2}$ \\
\hline Matadero . & SI & SI & SI & SI & SI \\
\hline Molino de Coronas & Si & SI & SI & NO & NO \\
\hline Molino del Rey & SI & SI & SI & $\mathrm{SI}$ & SI \\
\hline Molino del Matadero & SI & SI & NO & NO & NO \\
\hline Molino del Batán & SI & SI & SI & NO & NO \\
\hline Casa Parador & SI & SI & SI & NO & SI \\
\hline Casa de Comedias & NO & $\mathrm{SI}$ & NO & SI & SI \\
\hline Hornos de hilar seda & SI & NO & NO & NO & NO \\
\hline Diversas casas en la ciudad ${ }^{3}$ & SI & SI & SI & SI & SI \\
\hline Tierras de los Alcázares & SI & SI & SI & SI & SI \\
\hline
\end{tabular}

Hemos agrupado en este apartado aquellos propios que podrían ser considerados bienes inmuebles.

2 La Encañizada se perdió en un juicio en 1819.

${ }^{3}$ El número de casas varía según el año: a principios de siglo habia más de 15 . En 1820 eran 40 casas. 


\begin{tabular}{|c|c|c|c|c|c|c|}
\hline & CONCEPTO & 1700 & 1744 & 1760 & 1799 & 1820 \\
\hline & Siete tahullas en Molino del Rey ........ & NO & NO & NO & SI & SI \\
\hline & Tahullas en Santa Cruz y San Benito ... & NO & NO & NO & SI & SI \\
\hline & Millares del Campo ... & NO & SI & SI & SI & $\mathrm{SI}$ \\
\hline & Hierbas de Fortuna y demás & SI & SI & SI & SI & SI \\
\hline & Bóvedas (Son seis) & SI & SI & SI & SI & SI \\
\hline B) & PROPIOS MENORES & & & & & \\
\hline & Puestos de pan & SI & SI & NO & NO & $\mathrm{SI}$ \\
\hline & Puestos herrador & SI & SI & SI & NO & NO \\
\hline & Almacenes de pescado .. & SI & SI & SI & SI & $\mathrm{SI}$ \\
\hline & Títulos de escribanos ${ }^{4}$ & SI & SI & SI & SI & SI \\
\hline & Fábrica de pólvora & NO & SI & NO & NO & NO \\
\hline & Sosa de pinatar $\ldots \ldots \ldots$ & NO & SI & SI & NO & NO \\
\hline & Rejas pescadería & NO & SI & SI & SI & NO \\
\hline & Rejas carniceria & NO & SI & $\mathrm{SI}$ & SI & SI \\
\hline & Casetas de Feria & NO & NO & $\mathrm{SI}$ & SI & NO \\
\hline C) & RENTAS Y DERECHOS, ABASTOS & & & & & \\
\hline & Renta del almudinaje ........ & SI & SI & SI & SI & SI \\
\hline & Renta del almotacenia & SI & SI & SI & SI & SI \\
\hline & Rentas de las penas de cámara & SI & SI & SI & SI & SI \\
\hline
\end{tabular}

${ }^{4}$ El número de escribanía en 1685 era el de seis. En 1760, dos y en 1799 sólo quedaba una escribanía. 
Aproximación al estudio de las haciendas locales bajo Carlos III

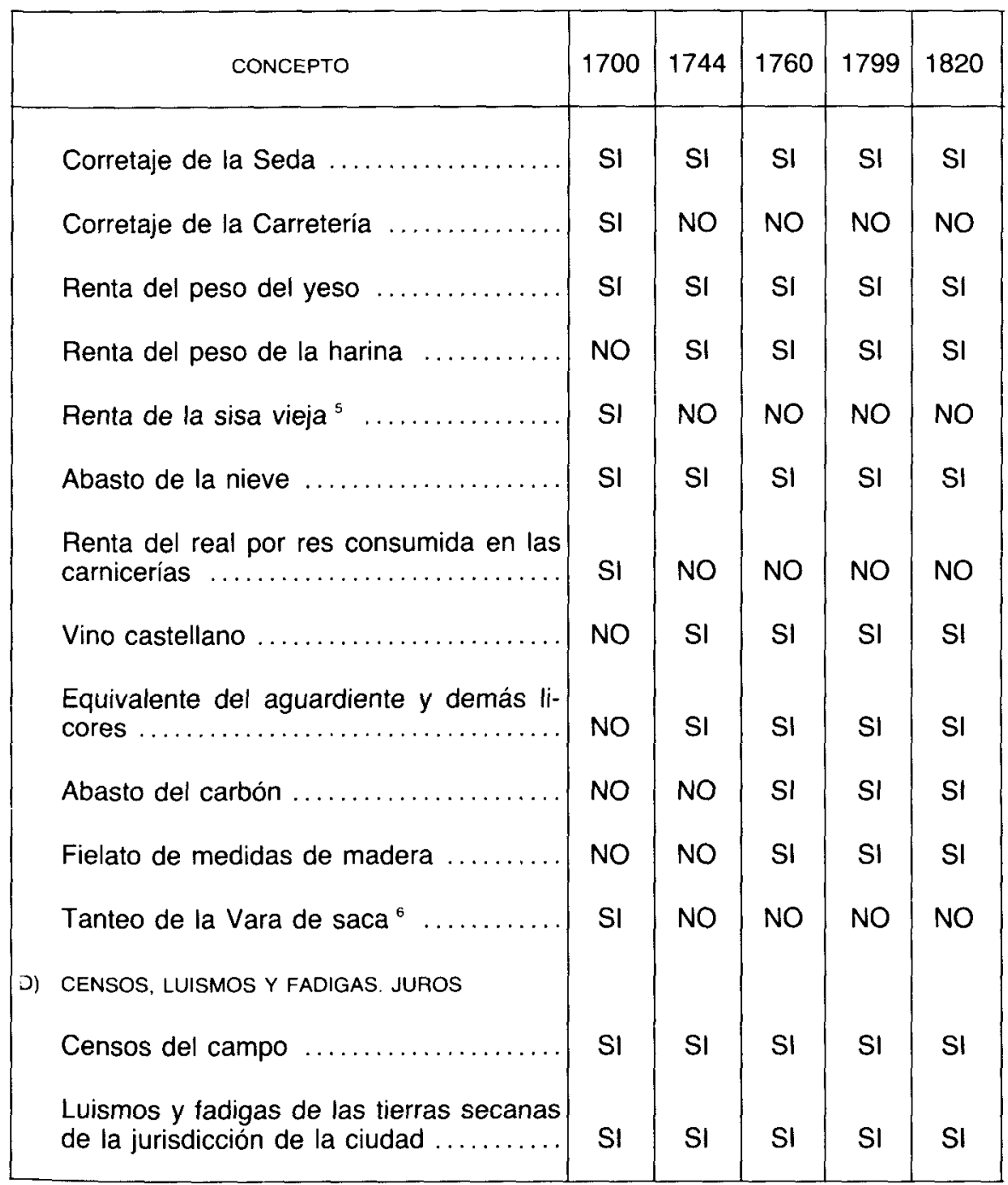

${ }^{5}$ La renta de sisa vieja se cobraba para reparación de caminos y obras públicas y consistía en 10 cuartos sobre cada galera de vino y $10 \mathrm{mrs}$. de cada carga y $8 \mathrm{mrs}$ en cada cabrito.

- La vara de saca había estado en poder de Cartagena desde 1675 al 1688 , y estaba valorada en 12.000 reales por año. Sobre ella pesaba un censo. 


\begin{tabular}{|l|c|c|c|c|c|}
\hline \multicolumn{1}{|c|}{ CONCEPTO } & 1700 & 1744 & 1760 & 1799 & 1820 \\
\hline Juros ${ }^{7} \ldots \ldots \ldots \ldots \ldots \ldots \ldots \ldots \ldots . \ldots$ & $\mathrm{SI}$ & $\mathrm{SI}$ & $\mathrm{SI}$ & $\mathrm{SI}$ & $\mathrm{SI}$ \\
E) ARBITRIOS $^{8}$ & & & & & \\
Arbitrio de un real sobre arroba de vino & $\mathrm{NO}$ & $\mathrm{SI}$ & $\mathrm{SI}$ & $\mathrm{SI}$ & $\mathrm{SI}$ \\
Arbitrio de dos mrs. sobre libra de carne. & $\mathrm{NO}$ & $\mathrm{SI}$ & $\mathrm{SI}$ & $\mathrm{SI}$ & $\mathrm{SI}$ \\
Arbitrio sobre el estanco del aguardiente. & $\mathrm{NO}$ & $\mathrm{SI}$ & $\mathrm{SI}$ & $\mathrm{SI}$ & $\mathrm{SI}$ \\
\hline
\end{tabular}

Cuadro: elaboración propia.

La observación detenida del cuadro 1 nos ofrece la visión de una inmensa panoplia compuesta de elementos tan dispares como lo pueden ser la obtención de pescado en la Encañizada, allá por el Mar menor y a más de $50 \mathrm{~km}$ de la capital y las casas del matadero en la capital, o los molinos harineros, a orillas del Segura a su paso por la ciudad, etc. Quizás lo único que tenían en común fuera el que todos los propios incidian, de una forma $u$ otra, en la vida mercantil de Murcia y sus pedanías.

Merece especial atención la escasa importancia de la tierra entre los propios del Concejo murciano. Habría que estudiar, y por tanto remontarse cinco siglos atras, la influencia de los repartimientos de Murcia, tras su reconquista, en el patrimonio de su concejo pues a lo largo del siglo XVIII sólo tenemos constancia de un mote de 32 fanegas de tierras de secano en el término de los Alcázares, que ya en el 1685 rentaban 150 reales de vellón y que, al cabo de un siglo, aparecian arrendados en 450 reales de vellón por año.

También podemos observar en este cuadro 1 cómo los propios han sufrido una ligera variación a lo largo del siglo XVIII, aunque los propios desaparecidos o incorporados a la lista no significan gran cosa dentro del contexto general de los propios del Ayuntamiento de Murcia.

7 En 1685-1700 el juro era de 200 ducados sobre las alcabalas de la ciudad.

${ }^{8}$ Estos arbitrios perduraron a lo largo del siglo. Para más información remitimos al capítulo "Arbitrios". 
¿Qué ingresos proporcionaban estos propios a las arcas municipales? ¿Cuál era su importancia respecto a los arbitrios? El cuadro 2 nos va a servir para encontrar las posibles respuestas. Los ingresos de los propios, así como los procedentes de los arbitrios apenas sufrieron variación bajo el reinado de Carlos III, sobre todo desde la publicación del reglamento de 1760 , manteniéndose en torno a los 350.000 reales los ingresos procedentes de propios y entre los 115.000 y 120.000 reales los procedentes de los arbitrios. Al final de siglo apreciamos un fuerte descenso en las cantidades obtenidas de propios, en tanto que los productos de los arbitrios experimentan un aumento del 30 por 100, pasando de los 120.000 reales a más de 150.000 reales (véase el cuadro 2).

Los diferentes componentes de propios tenían, asimismo, ingresos acordes a su importancia dentro del contexto económico general. Así, el matadero, el equivalente de aguardiente y el abasto de nieve eran los que ocupaban los primeros lugares en importancia de ingresos para el Ayuntamiento de Murcia. La encañizada y los molinos harineros les iban a la zaga. La breve extensión de este artículo nos impide desarrollar pormenorizadamente cada uno de los apartados de propios y sus valores, y sólo hemos seleccionado el propio de la Encañizada y los ingresos obtenidos por los censos (ver cuadros 3 y 4).

CUADRO 2. RENTAS PROCEDENTES DE PROPIOS Y ARBITRIOS DE LA CIUDAD DE MURCIA

\begin{tabular}{|c|c|c|c|}
\hline AÑO & $\begin{array}{c}\text { PRODUCTO DE } \\
\text { PROPIOS }\end{array}$ & $\begin{array}{c}\text { PRODUCTO DE } \\
\text { ARBITRIOS }\end{array}$ & TOTALES \\
\hline 1759 & 348.546 reales & 119.944 reales & 468.490 reales \\
1760 & 353.667 reales & 115.375 reales & 469.042 reales \\
1762 & 353.667 reales & 115.375 reales & 469.042 reales \\
1764 & 348.944 reales & 119.852 reales & 468.796 reales \\
$179{ }^{*}$ & 232.470 reales & 153.942 reales & 386.412 reales \\
1820 & 274.844 reales & - & - \\
\hline
\end{tabular}

* En este año se vendieron 21 casas pertenecientes a propios y sus capitales fueron impuestos en el fondo de Administración. 


\section{CUADRO 3}

CENSOS, LUISMOS Y FADIGAS DEL AYUNTAMIENTO DE MURCIA

(1755-1769)

(Producto en reales de vellón)

\begin{tabular}{|c|r|r|r|r|}
\hline AÑO & CENSO & $\begin{array}{r}\text { LUISMOS } \\
\text { Y FADIGAS }\end{array}$ & TOTAL & $\begin{array}{r}\text { VALOR DEL } \\
7 \text { por } 100\end{array}$ \\
\hline 1755 & 1.705 & 3.720 & 5.425 & 379 \\
1756 & 5.440 & 9.641 & 15.081 & 1.055 \\
1757 & 4.522 & 7.697 & 12.219 & 855 \\
1758 & 2.659 & 10.808 & 13.467 & 942 \\
1759 & 2.685 & 7.428 & 10.113 & 707 \\
1760 & 2.518 & 5.139 & 7.657 & 535 \\
1761 & 2.769 & 22.135 & 24.904 & 1.743 \\
1762 & 2.005 & 5.315 & 7.320 & 512 \\
1763 & 852 & 5.888 & 6.740 & 471 \\
1764 & 4.171 & 8.764 & 12.936 & 905 \\
1765 & 5.064 & 3.348 & 8.413 & 588 \\
1766 & 1.774 & 16.589 & 18.363 & 1.285 \\
1767 & 3.743 & 5.067 & 8.810 & 616 \\
1768 & 4.683 & 1.757 & 6.441 & 450 \\
1769 & 4.425 & 1.590 & 6.015 & 421 \\
\hline \multirow{2}{*}{$170 T$ TOLES } & 49.019 & 114.892 & 163.912 & 11.473 \\
\hline
\end{tabular}

Nota: El valor de 7 por 100 corresponde a los gastos por administración por lo que la cantidad final resultante liquidada en Depositaría asciende a la cantidad de 152.439 reales de vellón (ciento cincuenta y dos mil cuatrocientos treinta y nueve).

Cuadro: elaboración propia. 


\section{CUADRO 4}

PRODUCTOS DEL PROPIO DE LA ENCAÑIZADA DEL MAR MENOR

(1764-1780)

\begin{tabular}{|c|c|c|}
\hline ANOO & $\begin{array}{c}\text { PRODUCTO POR } \\
\text { ADMINISTRACION }\end{array}$ & $\begin{array}{c}\text { PRODUCTO POR } \\
\text { ARRENDAMIENTO }\end{array}$ \\
\hline 1764 & 21.859 rls.v. & - \\
1765 & - & 18.000 rls.v. \\
1766 & - & - \\
1767 & - & 13.333 rls.v. \\
1768 & - & 13.333 rls.v. \\
1769 & - & 13.333 rls.v. \\
1770 & - & 13.333 rls.v. \\
1771 & - & 13.333 rls.v. \\
1772 & - & 13.333 rls.v. \\
1773 & - & 25.000 rls.v. \\
1774 & - & 29.687 rls.v. \\
1775 & - & 38.532 rls.v. \\
1776 & - & 19.000 rls.v. \\
1777 & - & 19.000 rls.v. \\
1778 & - & 19.000 rls.v. \\
1779 & - & 12.000 rls.v. \\
1780 & - & 12.000 rls.v. \\
\hline & 21.859 rls.v. & 272.039 rls.v \\
\hline
\end{tabular}

Fuente: A.H.M.N. Legajo n. ${ }^{\circ} 3901$. 


\section{CUADRO 5. RELACIÓN DE CENSOS QUE EL AYUNTAMIENTO DE MURCIA PAGABA DE SUS PROPIOS (año 1757)}

\section{Beneficiario}

1. Convento de San Francisco

2. Catedral de Murcia. Capellanía que fundó el canónigo Macias Coque ...

3. Convento de Santo Domingo

4. Convento-Hospital de Ntra. Sra. de Gracia .....

5. Beneficiados de la Catedral .....................

6. Cofradía de la Purísima Concepción

7. Pedro Forcada

8. Pedro Saavedra y Fajardo, rexidor

9. Francisca Saurin Gallego

10. Pias Fundaciones ........

11. Marqués de Corvera .....

\section{Cantidad-Causa del censo}

12 reales por misas.

390 reales por un principal de 1.200 ducados que se tomó sobre unas casas en la Parroquia de Sta. Catalina para ensanche de la carniceria mayor de la ciudad.

495 reales

90 reales por un valor de principal de 3.000 reales sobre una casa sobre la que se asienta la Escribania Mayor del Ayuntamiento.

99 reales por un principal de 3.200 reales sobre la casa de Comedias.

275 reales por un principal que pesaba sobre las Rejas de la Pescadería.

99 reales por un principal sobre los Propios.

660 reales por un principal de 2.000 ducados que pesaba sobre las carnicerías de la ciudad.

1.980 reales por dos principales: uno de 4.000 ducados de vellón que se dieron para la construcción de la Casa Parador; otro, de 2.000 ducados para la apertura del cauce del Azarbe Mayor que corre por Monteagudo (ambos se habían impuesto en el siglo $x V I)$.

4.500 reales por un principal de 150.000 reales que se habian impuesto sobre los propios y rentas.

1.440 reales por un principal de 48.016 reales que quedo de resto de los 12.000 ducados en que se tanteó la vara de Alcalde Mayor de Sacas.

Fuente: A.M.M., leg. 3085. 
Todos estos ingresos servian para que el Concejo Murciano pagara los gastos y sueldos de su personal adscrito, los gastos del cabildo por asistencia a ceremonias litúrgicas y celebración de fiestas, los gastos de reparaciones de caminos de la huerta y monda de las acequias, limpieza de las calles de la ciudad, a la curación de presos enfermos de la Cárcel Real; también salian de los propios los gastos de la construcción del nuevo puente sobre el río Segura, mientras durase su realización, los repartimientos de utensilios y paja -sólo en determinados años-, etc.

En el Reglamento de 1760 para la Ciudad de Murcia, se señalan los ingresos por propios y arbitrios en la cantidad de 469.042 reales de vellón por año y unos gastos - cargas, según la época- que ascienden a la cantidad de 294.220 reales, sobre un presupuesto de contingentación de 333.665 reales. Estas cantidades arrojaban un superávit de 174.820 reales, en un caso, o de 135.377 reales en otro. Este sobrante encontraría pronto destino: en 1767, el Consejo de Castilla, y por acuerdo del 19 de mayo y circular del 23 , dictaminará la redención de censos sobre los propios con las dos terceras partes de los sobrantes de los mismos ${ }^{15}$. A partir de este momento la suerte de los censos aplicados a los propios y arbitrios de la ciudad estaba echada. Estas disposiciones de Carlos III, recordadas posteriormente por su hijo Carlos IV, iban destinadas a la redención total de los censos perpetuos. En 1803 volvemos a encontrar leyes para la libre imposición de censos, pero con la condición de ser redimibles al 3 por $100^{16}$.

El sistema preferido por el Ayuntamiento de Murcia, para la gestión de sus propios y arbitrios era la del arrendamiento. Preferentemente el arriendo se hacia por una duración temporal que oscilaba entre los tres y cuatro años, según peculiaridad del propio. Hubo circunstancias que obligaron a arrendar algunos propios por un tiempo de ocho años ${ }^{17}$. El acto de arrendamiento se realizaba mediante subasta pública, y a la cual los señores regidores estaban excluidos de participar en la misma ${ }^{18}$. El acto de la subasta era público y el propio se adjudicaba al mejor postor. Los arrendata-

15 Nov. Rec., XIV

${ }^{16}$ Nov. Rec., XXIII, Resolución 18 de noviembre de 1803.

17 Este fue el caso del Abasto de la Nieve. Murcia no es una región propicia para la caida de nieve frecuente.

${ }^{16}$ Nov. Rec., VII, 16, 4, 13 y 27, y Nov. Rec. VII, $9,7$. 
rios debían de ofrecer fianzas en sus posturas que sobrepasaran el valor del propio ofertado como garantía de su gestión arrendaticia ${ }^{19}$. A partir del Reglamento de 1760, y por mandato del Consejo de Castilla, los arriendos no podian ser superiores en tiempo a un año, y cualquier otro plazo solicitado llevaba consigo la consulta obligada del Consejo, que el que finalmente aprobaba la concesión del arriendo ${ }^{20}$.

Sobre el arrendador o arrendatario -que así aparece en sus dos formas- estaba la Junta Municipal de Propios y Arbitrios del Ayuntamiento de Murcia. Su antecedente se encuentra en la Instrucción de 1745 sobre Arbitrios, pero su configuración definitiva no aparecerá hasta la Instrucción de $1760^{21}$. El Intendente presidía dicha Junta y estaba acompañado de dos regidores, un mayordomo, un contador $y$, desde su creación, de los diputados del Común y el personero síndico. El Reglamento para la ciudad de Murcia, que fue aprobado por el Consejo mediante Decreto del 9 de marzo de 1762, responsabiliza a los miembros de la Junta, mediante garantía de bienes propios, de la observancia exacta del cumplimiento del Reglamento uen todas sus partes sin alteración alguna» ${ }^{22}$. Esta responsabilidad se extendia desde la recaudación de los caudales hasta su reparto; el nombramiento del mayordomo también era acometido por la Junta: «... pertenece a su conocimiento privado". La Junta estaba configurada como un órgano asesor, fiscalizador del uso de los caudales procedentes de los propios. A veces, y según hemos podido comprobar en los libros de Actas de la Junta, su actuación le llevaba a enfrentarse al mismo ayuntamiento. El libro de Actas de las reuniones de la Junta lo llevaba un escribano del Ayuntamiento y en el mismo se asentaban, además de las aprobaciones de las partidas para satisfacer las necesidades del Concejo de la ciudad de Murcia, las disposiciones que venían dictadas desde la Contaduría General de Propios, así como las que derivaban de la propia Intendencia.

Conocemos el nombre de los componentes de la Junta, pues en el Archivo Histórico de la ciudad de Murcia hemos encontrado casi todos

${ }^{19}$ Reglamento de Propios y Arbitrios para la Ciudad de Murcia. Año 1762. A.H.M.M., leg. 1534.

${ }_{20}$ En noviembre de 1769, el consejo de Castilla, a propuesta del Contador General de Propios y Arbitrios, Don Manuel Becerra, llama la atención a la Junta Municipal de Propios de Murcia por haber arrendado el abasto de nieve y agua fría por un período de ocho años y no hacerlo por un año, como estaba ordenado.

${ }^{21}$ Nov. Rec. VII, 13, cap. 13.

${ }^{22}$ Reglamento de Propios... Esta observación aparece en lo que podriamos llamar el epílogo a dicho Reglamento, y parece ser una obsenvación del mismo Becerra. 
los libros de Juntas correspondientes a la vida de la misma, desde su creación. Cuando el Intendente no podía asistir a las sesiones de la Junta, ocupaba su lugar el Contador provincial, que también solía prestar su asistencia a las sesiones que la Junta le requiriera. El funcionamiento de la Junta venía determinado por el acuerdo municipal sobre algún gasto, ordenando su pago, siempre que correspondiese a una partida de las reseñadas en el Reglamento. La Junta, una vez analizada la petición concejil, libraba los capitales correspondientes. Si era preciso un gasto extraordinario, no especificado en el Reglamento, su libramiento debía de estar autorizado por Madrid.

Hemos constatado que además de esta Junta municipal de Propios y Arbitrios existían otras Juntas paralelas, sobre todo de aquellos propios que por su estructura requerían un control más severo de su funcionamiento. Nos estamos refiriendo a la Junta del Pósito y a la Junta del Abasto de nieve. De la primera, hemos encontrado libros de Juntas desde 1763 a $1803^{23}$; de la segunda, apenas tenemos vagas referencias, que aunque nos confirman su existencia, no son tan relevantes como la anterior.

Por último, sólo nos queda por hablar del Reglamento de Propios y arbitrios de la Ciudad de Murcia ${ }^{24}$. Este Reglamento, del que ya hemos dado algunas noticias, es más que una norma de obtención de ingresos municipales, una sistemática exposición de las cargas y gastos “... que corresponden a los propios y arbitrios de la Ciudad de Murcia y a que debe ceñirse precisamente la Junta establecida para la administración de ellos". Su prólogo alude a las fuentes que inspiran la relación de las diferentes partidas. Así, en lo tocante a propios son las certificaciones del Contador de la Ciudad de Murcia, Don Mateo Tomás de Jumilla, en tanto que para los arbitrios se contaba con las certificaciones de Don José Ceballos, Contador principal de la Intendencia y Superintendencia del Reino de Murcia. Después, sigue una relación de los propios y rentas que goza la ciudad de Murcia, sin especificar los ingresos potenciales de cada una de ellos en particular, y sí aparece al final de dicha relación el total producido anualmente por los mismos; en cuanto a los arbitrios sucede lo mismo, salvo que aquí se indica la fecha de las reales facultades

${ }^{23}$ A.H.M.M., leg. 2700, 2701, 2702 y 2704.

${ }^{24}$ Lamentamos que por el carácter de este artículo no podamos ofrecer el texto íntegro del Reglamento, por lo que nos quedamos con la duda de que si el resumen que hemos presentado cumple los objetivos propuestos de acercar al lector a su conocimiento. 
que autorizaban a la ciudad de Murcia a imponerlos. Ambos conceptos, propios y arbitrios, producían una renta de 469.042 reales por año.

Más adelante aparece la descripción de las cargas y la distribución de los capitales obtenidos por las rentas de propios y arbitrios, siguiendo un esquema prefijado en base a las partidas que resultan de las certificaciones, las que se abonan por dotación fija y anual para gastos ciertos y las partidas que se abonan para extraordinarios. Aquí podemos apreciar hasta el más pequeño detalle de cada una de las cargas, empezando por los censos, de los que hemos contabilizado hasta 22 censos - algunos con facultad Real—, siguiendo por los gastos por fiestas religiosas (¡Ocho!, contabilizadas). Los salarios vienen a continuación empezando por el Sr. Corregidor de la Ciudad, al que se le asignan 6.600 reales/ año, y los dos Alcaldes mayores de lo civil y criminal de la Ciudad y que cobran "por razón de asesorías en los pleitos y dependencias de su Ayuntamiento» 250 reales cada uno y por año. Los 33 Regidores que contaba el Ayuntamiento en estos años tenían de salario anual 4.000 reales, los cuales se les libraba "por costumbre inmemorial». Así podríamos exponer cada uno de los casi 40 empleados directos que sostenia el Ayuntamiento. Por último, aparecen las cargas por pensiones a viudas de algún antiguo personal, y otras varias que se escapan de la finalidad de este artículo su pormenorización y exposición. Cabe resaltar que este Reglamento elimina como gasto fijo algunas cargas que aparecian en las certificaciones de años anteriores, como por ejemplo el correspondiente a la que autorizaba el pago de 117 reales al que sirve la suerte de sellar madera y al que ostentaba, igualmente, la suerte del Sello y Pendón que venía cobrando de tiempo «inmemorial», o aquella otra certificación del Comisario del Contraste, etc..., etc...

El Reglamento acaba con un resumen de los ingresos y gastos, el sobrante resultante y algunas anotaciones a ciertos errores en algunas certificaciones, asi como las indicaciones y consejos a la Junta sobre las responsabilidades de la misma en la gestión de los caudales procedentes de los propios de la ciudad de Murcia. 